EXTENDED REPORT

\title{
Topography of the frequency doubling perimetry visual field compared with that of short wavelength and achromatic automated perimetry visual fields
}

\author{
J Landers, A Sharma, I Goldberg, S Graham
}

Br J Ophthalmol 2006;90:70-74. doi: 10.1136/bjo.2005.071266

See end of article for authors' affiliations

\section{Correspondence to:} Dr John Landers, Park House, Floor 4, Suite 2 , 187 Macquarie Street, Sydney, NSW 2000 Australia; john.landers@ bigpond.com

Accepted for publication 1 August 2005
Background: Traquair described the topography of visual field sensitivity as a "hill" or "island" of vision. Achromatic automated perimetry (AAP) demonstrates this shape of the visual field in photopic conditions. Techniques claimed to target the magnocellular pathway (frequency doubling perimetry, FDP) and those using a stimulus targeting the koniocellular pathway (short wavelength (or blue on yellow) automated perimetry, SWAP), might produce one that is different. The authors compared the visual field topography from FDP with those of SWAP and AAP, to investigate whether there were significant differences in their shape.

Method: A sample of 51 patients with previously confirmed normal perimetry were recruited; either low risk glaucoma suspects or normal controls. AAP, SWAP, and FDP perimetry was performed in random order on the same day. The topography of each field was analysed to determine its average shape and to compare results in the same individuals.

Results: The topography of the visual field produced by each perimeter differed significantly. While all three had maximal sensitivity centrally, over the 24 degrees from the centre to the periphery, mean sensitivities decreased by 4.9 decibels (dB) for AAP and 7.3 dB for SWAP, while FDP sensitivities by just $1.8 \mathrm{~dB}$ over 20 degrees (the extent of the FDP field). FDP mean sensitivities decreased by approximately $0.3 \mathrm{~dB}$ with every 10 year increase in age, compared with $1 \mathrm{~dB}$ for $A A P$ and $2 \mathrm{~dB}$ for SWAP.

Conclusion: While the topography of the SWAP (koniocellular) field is steeper than corresponding AAP fields, that of the FDP (magnocellular) visual field was considerably flatter. The difference in this shape may reflect retinotopic or cortical mechanisms, which are specific to the magnocellular pathways.
$\mathrm{T}$ he topography of the visual field has been the basis of the functional assessment of the visual pathways for more than 150 years. ${ }^{1}$ It is derived from the differential light threshold at various locations in the visual field. ${ }^{2}$ In photopic circumstances, it is more sensitive centrally, gradually falling with increasing eccentricity. ${ }^{3}$ Traquair described it as an "island" or a "hill" of vision in a sea of blindness. ${ }^{5}$ Among other influences including stimulus size and background luminance, this shape is a function of the relative densities of retinal ganglion cells and spatial summation at various retinal eccentricities. ${ }^{6}$

By convention, the stimulus used to plot the topography of the visual field is an achromatic point of light on a background of 31.5 apostilbs: it is not specific to particular ganglion cell lines. ${ }^{7}$ The final shape of the "hill of vision" produced by achromatic perimetry may be determined by the stimulation of those ganglion cells that are in the majoritynamely, the parvocellular line. ${ }^{8}$

Short wavelength automated perimetry (SWAP) uses a yellow background and a blue target stimulus. It targets the koniocellular pathway projecting from "blue on" cells in the retina, which have different temporal and spatial properties from the parvocellular pathway. Therefore, the topography of this visual field may be different from that produced by an achromatic stimulus such as achromatic automated perimetry (AAP). ${ }^{9-11}$

Frequency doubling perimetry (FDP) utilises a test stimulus made up of alternating light and dark lines, with low spatial frequency and high temporal frequency. ${ }^{12}{ }^{13}$ These parameters are claimed to stimulate selectively the magnocellular pathway, ${ }^{14}{ }^{15}$ and possibly a non-linear subset of this pathway (the My subset), ${ }^{16}{ }^{17}$ although evidence for this subset is controversial in humans. ${ }^{14}{ }^{15}$ Because the magnocellular ganglion cells are distributed differently from parvocellular, visual field topography produced by FDP may again differ from that seen with AAP. ${ }^{18}$

This study was undertaken to examine the topography of the FDP visual field and then compare it with those produced by SWAP and AAP techniques that have been described previously, ${ }^{9-11}{ }^{19-22}$ to investigate whether or not there are significant differences in their shape.

\section{METHOD}

We recruited a consecutive sample of normal subjects $(n=15)$ and low risk glaucoma suspects $(n=36)$ with normal visual fields from those patients who were regularly attending an urban glaucoma clinic. Exclusion criteria were corrected visual acuity worse than 6/9, the presence of definite glaucomatous optic neuropathy, ${ }^{23}$ diabetes, cataract, refractive error of more than $5 \mathrm{D}$ sphere or $3 \mathrm{D}$ of cylinder, pupil size less than $3 \mathrm{~mm}$ and/or corneal or retinal disease that could affect test results. The glaucoma suspect group had a family history of glaucoma, or suspicious optic discs with no definite structural changes ${ }^{23}$ and normal intraocular pressure (IOP $<21 \mathrm{~mm} \mathrm{Hg}$ on at least three occasions), or ocular hypertension (IOP $\geqslant 21 \mathrm{mmHg}$ on at least three occasions). They were not high risk or "pre-perimetric" suspects, having previously been found to have normal SWAP and FDP visual fields during the preceding 2 years. All

Abbreviations: $A A P$, achromatic automated perimetry; FDP, frequency doubling perimetry; GHT, glaucoma hemifield test; IOP, intraocular pressure; PSD, pattern standard defect; SWAP, short wavelength automated perimetry 


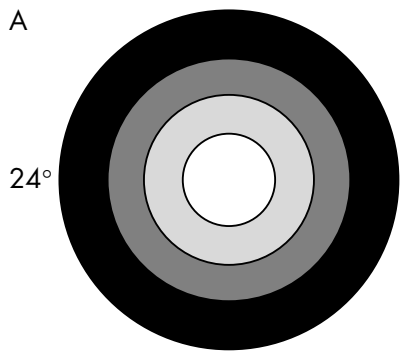

Figure 1 The position of visual field zones for (A) the Humphrey field analyser for AAP and SWAP and (B) FDP.

B

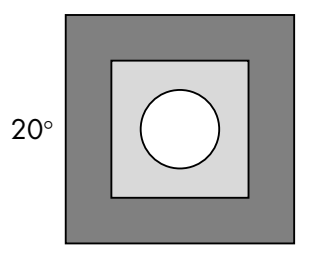

Zone $1\left(10^{\circ}\right.$ eccentricity)

Zone 2 (15 eccentricity)

Zone $3\left(20^{\circ}\right.$ eccentricity)

Zone 4 (24 eccentricity)

patients also had at least two consecutive normal visual fields on threshold AAP testing performed within the preceding 2 years, so they were experienced at perimetry. One eye from each patient was considered. When both eyes were eligible, a random choice was made.

AAP, SWAP, and FDP were performed in random order after obtaining informed consent in accordance with the South Eastern Sydney Area Health Services clinical research ethics committee requirements. AAP was performed using the Humphrey field analyser II (HFA) (Carl Zeiss, Dublin, CA, USA) with a 24-2 SITA-Standard program. SWAP was performed using the HFA with a 24-2 SWAP full threshold program, in which the background was illuminated with a yellow light at $100 \mathrm{~cd} / \mathrm{m}^{2}$ and the target was a blue stimulus (440 nm), size V (1.72 degrees). Subjects had the same refractive correction for both tests. A normal field was defined as a field with a normal glaucoma hemifield test (GHT) and a mean defect and a pattern standard defect

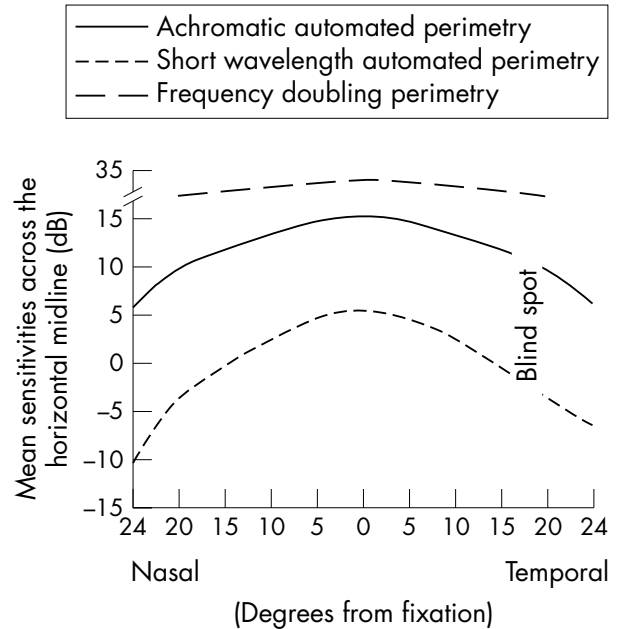

Figure 2 Mean visual field sensitivities, averaged from either side of the horizontal midline of the visual field for AAP, SWAP, and FDP. (Sensitivities for AAP and SWAP have been converted to contrast decibels in order to be comparable with FDP.)
(PSD) of $\mathrm{p}>5 \%$. A normal field was also one in which there were less than five points whose sensitivities were no worse than $\mathrm{p}<5 \%$, with no clustering of such points. FDP was performed using a Humphrey-Zeiss frequency doubling technology (FDT) perimeter (Carl Zeiss, Dublin, CA, USA) using a full threshold N-30 program. A normal visual field had a mean defect and a PSD of $\mathrm{p}>5 \%$ and had no more than two target locations with sensitivities no worse than $\mathrm{p}<5 \%$, with no clustering of locations. In all cases, fields were considered reliable if there were less than $33 \%$ false negative and false positive errors and less than $20 \%$ fixation losses.

The central 24 degrees of the HFA and the central 20 degrees of the FDP field were considered for analysis. Each test point in a field was assigned to a particular zone, depending on its eccentricity (fig l) and a particular quadrant depending on location. Summary statistics (mean and standard deviation (SD)) were calculated for each zone and quadrant. The rate of change in these summary statistics with increasing eccentricity was then calculated and compared between tests. Because AAP and SWAP are measured in differential light sensitivity decibels and FDP is measured in contrast decibels they are not directly comparable. For calculations and figures in which they were compared, AAP and SWAP values were converted into contrast decibels ${ }^{24}$ :

Contrast decibels $=-20 \log \left(10^{((\mathrm{DLSdB}-40) /-10)} / 31.5\right)$

where DLSdB $=$ differential light sensitivity decibels.

Statistical Analysis System 6.12 (SAS Institute Inc, Cary, NC, USA) was used for statistical analysis including descriptive statistics, unpaired two tailed Student's $t$ test and simple linear regressions. In linear regressions age and visual field sensitivity were used as continuous variables and visual field zone was used as a categorical variable. Regression coefficients, test statistics, and $\mathrm{p}$ values were presented.

\section{RESULTS}

There were 51 patients in our sample; 23 males (45\%) and 28 females (55\%). Average age was 55 (SD 13) years: 55 (12) years for males and 55 (14) years for females $(t=0.00$; $\mathrm{p}=1.0)$. There were 15 normal patients $(29 \%)$ and 36 glaucoma suspects $(71 \%)$. There were no significant differences in mean defect between normal patients and glaucoma suspects for any of the tests $(t=0.97 ; \mathrm{p}=0.34$ for AAP, $t=0.54 ; \mathrm{p}=0.59$ for SWAP, and $t=0.52 ; \mathrm{p}=0.61$ for FDP). When a visual field was considered as a series of concentric zones (four for HFA and three for FDP) (fig 1), the mean

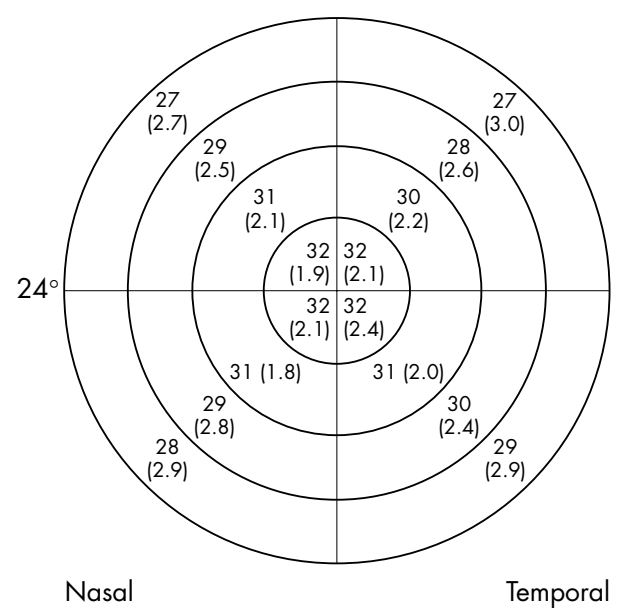

Figure 3 Mean visual field sensitivities in decibels (SD) for each quadrant of each zone for AAP using the Humphrey field analyser. (Eccentricity of visual field is indicated.) (Mean sensitivities in differential light sensitivity decibels.) 
sensitivities in each zone for all three methods showed a statistically significant decrease with increasing eccentricity: for AAP $\left(r^{2}=0.35, t=10.41 ; \mathrm{p}<0.0001\right)$, for SWAP $\left(r^{2}=0.32\right.$, $t=9.84 ; \mathrm{p}<0.0001)$, and for FDP $r^{2}=0.04, \quad t=2.33$; $p=0.021)$. However, the slope of the decrease in mean sensitivities differed considerably, with AAP mean sensitivities decreasing by $4.9 \mathrm{~dB}$ compared with SWAP mean sensitivities decreasing by $7.3 \mathrm{~dB}(t=15.32 ; \mathrm{p}<0.0001)$ over the 24 degrees from the centre to the periphery, compared with $1.8 \mathrm{~dB}$ over the 20 degrees of the FDP field $(t=49.07$; $\mathrm{p}<0.0001$ ) (fig 2). When the fields were divided into quadrants, the mean sensitivities for each zone within the quadrant were calculated (figs 3-5). There were similar decreases in the mean sensitivities for each quadrant zone with increasing eccentricity (table 1). SWAP had the greatest rate of mean sensitivity decrease followed by AAP and then FDP (table 1).

While both AAP and SWAP mean sensitivities were associated with age, such that mean sensitivity decreased by $1 \mathrm{~dB}$ for every 10 year increase in age for AAP $\left(r^{2}=0.39\right.$, $t=5.21 ; \mathrm{p}<0.0001)$ and by $2 \mathrm{~dB}$ for SWAP $\left(r^{2}=0.38\right.$, $t=5.52 ; \mathrm{p}<0.0001)$, a similar association between FDP mean sensitivity and age was not found $(0.3 \mathrm{~dB}$ decrease for every 10 year increase in age $)\left(r^{2}=0.01, t=0.77 ; \mathrm{p}=0.45\right)$. The relations between mean sensitivities and eccentricity persisted following adjustment for age (table 1). For both AAP and SWAP, peripheral field mean sensitivities decreased more rapidly with increasing age compared with central locations (fig 6). FDP fields did not show this trend with eccentricity (fig 6).

\section{DISCUSSION}

An improved understanding of the magnocellular pathway's properties and functions might enhance our prediction of glaucomatous damage and thus our use of this pathway to detect the disease, or its progression. This may result from greater knowledge of its retinal distribution, which may be delineated by its sensitivity topography on functional testing.

Our study confirms previous work, ${ }^{19-22}$ which showed that AAP visual field mean sensitivities are highest centrally and gradually decrease towards the periphery, in an expected pattern (fig 2). SWAP fields also demonstrated a "hill" shaped topography (fig 2), as has been previously documented for perimetry targeting the koniocellular pathway and which has also been documented in other forms of perimetry that selectively target parvocellular cells. ${ }^{25} 26$ However,

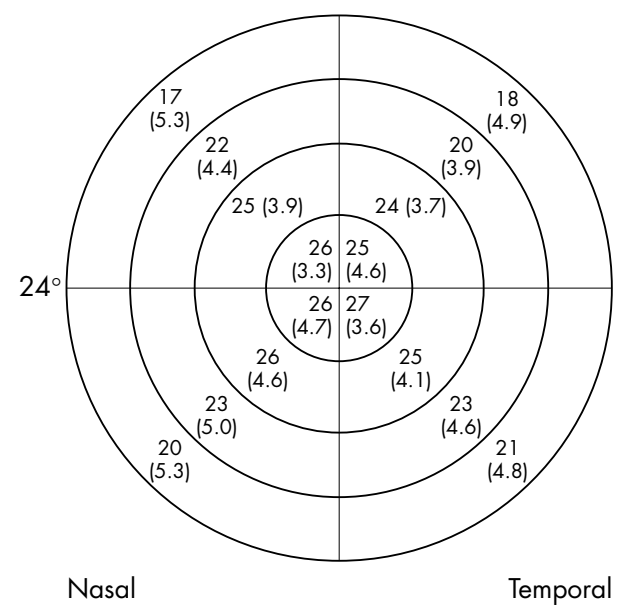

Figure 4 Mean visual field sensitivities in decibels (SD) for each quadrant of each zone for SWAP using the Humphrey field analyser. (Eccentricity of visual field is indicated.)

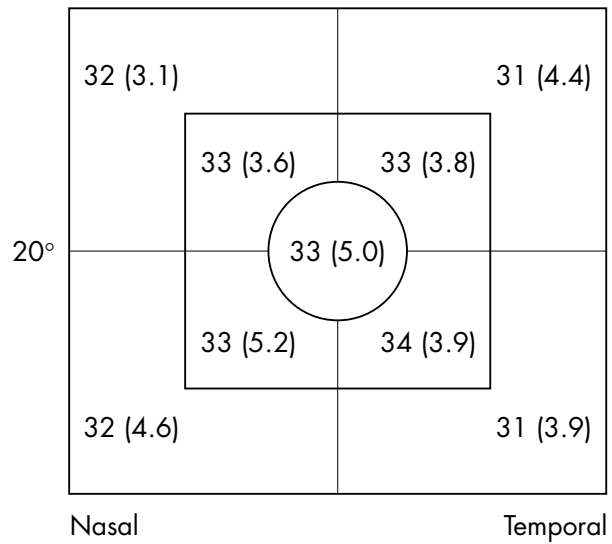

Figure 5 Mean visual field sensitivities in decibels (SD) for each quadrant of each zone for the FDP. (Eccentricity of visual field is indicated.) (Mean sensitivities in contrast decibels.)

although similar, the "hill" appeared much steeper among SWAP fields compared with AAP, with visual field sensitivity decreasing more rapidly with increasing eccentricity (fig 2 ).

By contrast, FDP fields had a considerably flatter topography than HFA fields (fig 2). Thus, they were different from that of Traquair's conventional island: a finding similar to motion perimetry, which targets the magnocellular pathway. ${ }^{27}$ This topography may be the result of the retinotopic distribution of retinal ganglion cells subserving the magnocellular pathway. While the proportion of parvocellular ganglion cells (midget cells) decreases with increasing eccentricity $^{2829}$ in a pattern similar to the shape of the conventional "hill of vision," the proportion of magnocellular ganglion cells (parasol cells) increases with increasing eccentricity, in line with its different visual functions, one of which is the detection of movement. ${ }^{28}{ }^{29}$

Cortical mechanisms may have a role in maintaining FDP sensitivities with eccentricity. ${ }^{14}$ At each visual field location, corresponding cortical cells in extrastriate areas might be arranged in a hierarchy of spatially frequency tuned channels. This hierarchy may ensure that at each point in the visual field there is a cortical channel maximally stimulated, thereby preventing any substantial reduction in visual field sensitivity with increasing eccentricity ( $\mathrm{T}$ Maddess, personal communication). Possibly one or both of these mechanisms may determine the observed shape of the FDP visual field, as may others including the possibility that it may be determined by spatial summation of the magnocellular pathway or the larger 10 degree targets used by the FDP. Further investigation should be done using the newer FDT Matrix visual field grid, which includes a larger number of tests zones similar to standard Humphrey visual fields. Since the test zones are smaller, however, the sensitivities may not follow the same pattern as the original FDP since with smaller test points, edge recognition becomes more of a factor.

HFA visual field sensitivities in this study decreased with increasing age while maintaining the same relation with eccentricity. ${ }^{30}$ SWAP field sensitivity decreased more rapidly with increasing age. ${ }^{30}$ In part this may be associated with increasing ocular media absorption with increasing age, although, Wild et al showed that even following correction for lens media absorption, SWAP sensitivity decreased at twice the rate of AAP sensitivity. ${ }^{30}$ Furthermore, both of these types of fields showed a more rapid decrease in mean sensitivity with age in the periphery compared with central locations. ${ }^{20} 30$ The slope of the field's "hill shape" thus increases with age. By contrast, unlike previous work, ${ }^{30}$ FDP mean sensitivities 
Table 1 Linear regression coefficients and test statistics for the relation between visual field mean sensitivities and increasing eccentricity

\begin{tabular}{|c|c|c|c|c|}
\hline \multirow[b]{2}{*}{ Quadrant } & \multicolumn{2}{|c|}{ Univariate analysis } & \multicolumn{2}{|c|}{ Multivariate analysis } \\
\hline & Coefficient & Test statistic & Coefficient & Test statistic \\
\hline \multicolumn{5}{|l|}{ SWAP } \\
\hline Superonasal & -2.90 & $10.72^{* \star \star *}$ & -2.90 & $12.09^{* \star * *}$ \\
\hline Superotemporal & -2.56 & $9.44^{* * * *}$ & -2.56 & $10.90^{* * * *}$ \\
\hline Inferonasal & -2.00 & $6.46^{* * * *}$ & -2.00 & $7.62^{* * * *}$ \\
\hline \multicolumn{5}{|l|}{ AAP } \\
\hline Superonasal & -1.57 & $10.74^{* * * *}$ & -1.54 & $11.98^{* \star * *}$ \\
\hline Superotemporal & -1.70 & $10.91^{\star \star \star *}$ & -1.65 & $12.10^{\star \star \star \star}$ \\
\hline Inferonasal & -1.54 & $10.12^{* * * *}$ & -1.50 & $11.10^{* * * *}$ \\
\hline $\begin{array}{l}\text { Inferotemporal } \\
\text { FDP }\end{array}$ & -1.20 & $7.89^{\star \star \star *}$ & -1.19 & $8.57^{* * * *}$ \\
\hline Superonasal & -0.52 & 1.32 & -0.47 & 1.14 \\
\hline Superotemporal & -1.16 & $2.66^{* *}$ & -1.11 & $2.35^{\star}$ \\
\hline Inferonasal & -0.80 & 1.63 & -0.70 & 1.32 \\
\hline Inferotemporal & -1.14 & $2.63^{* *}$ & -1.05 & $2.37^{*}$ \\
\hline
\end{tabular}

showed substantially less reduction with increasing age; this reduction did not reach statistical significance. FDP fields also showed no trend in mean sensitivity variation with age at different eccentricities. ${ }^{31}$

A limitation of our study is its relatively small sample size. However, it was large enough to detect a significant effect of eccentricity on visual field sensitivity and to detect the difference between this effect in HFA and FDP fields. Being clinic based, our sample may not represent the general population. It comprised a consecutive sample of normal patients and low risk glaucoma suspects with normal discs, AAP, SWAP and FDP. While it is possible some of them may have had some early visual pathway damage it is unlikely this
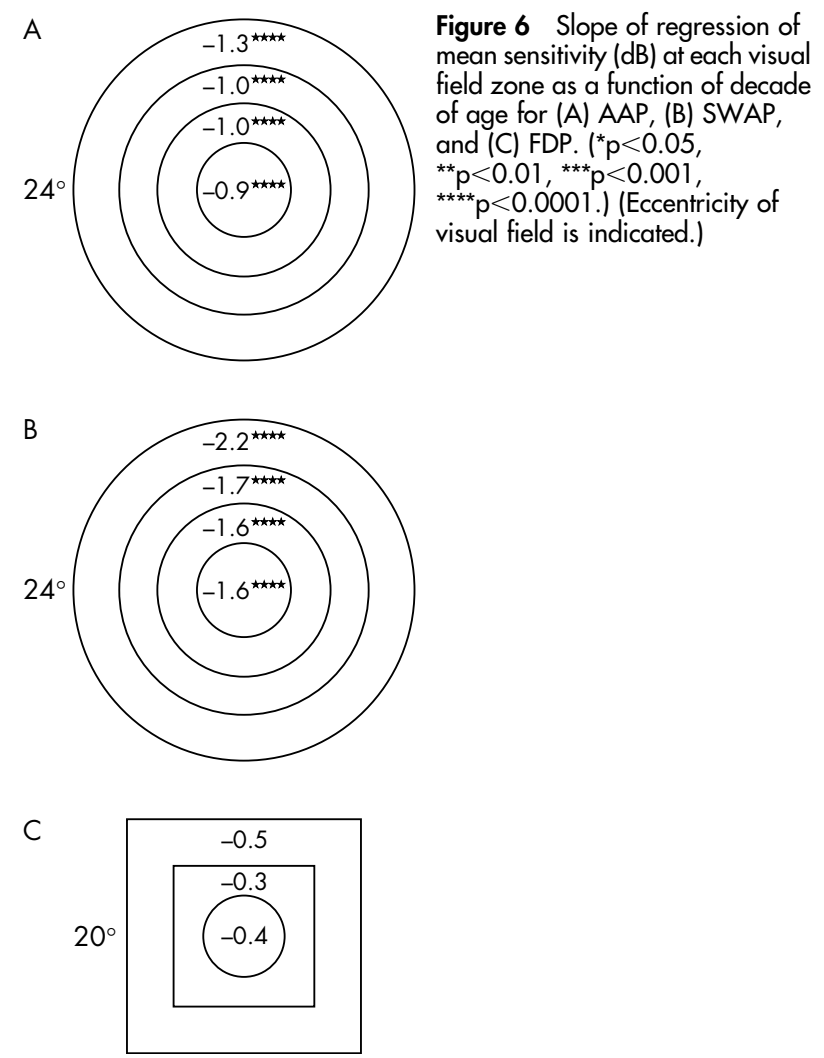

would have influenced the overall shape of hill of vision for a particular test type, but may have caused some localised reductions in some subjects.

In conclusion, compared with the topography of the SWAP (koniocellular) field, which is a "hill of vision" that was steeper than a corresponding AAP field, the FDP (magnocellular) visual field was considerably flatter than that described by the conventional "Island of Traquair." The difference in this shape may reflect retinotopic or cortical mechanisms, which are specific to the magnocellular pathway.

\section{ACKNOWLEDGEMENTS}

We thank associate professor Ted Maddess and the staff at Eye Associates for their support and assistance.

\section{Authors' affiliations}

J Landers, A Sharma, I Goldberg, S Graham, Eye Associates, Park House, Macquarie Street, Sydney, New South Wales, Australia

I Goldberg, Sydney Eye Hospital, University of Sydney, Sydney, New South Wales, Australia

I Goldberg, S Graham, Save Sight Institute, University of Sydney, Sydney, New South Wales, Australia

J Landers, Department of Medicine, University of Adelaide, Adelaide, South Australia, Australia

Financial support: This study was not subject to funding from any external source.

Financial disclosure: The authors have no proprietary or financial interest in any of the equipment used in this study.

Ethical approval: Ethical approval for this study was obtained from the South Eastern Sydney Area Health Services Clinical Research Ethics Committee.

\section{REFERENCES}

1 Kronfeld PC. The history of glaucoma. In: Tasman W, Jaeger EA, eds. Duane's clinical ophthalmology. Revised ed. Philadelphia: JB Lippincott, 1993;3, chapter 41.

2 Shapley R, Enroth-Cugell C. Visual adaptation and retinal gain controls. In: Osborne NN, Chader GJ, eds. Progress in retinal research. Oxford: Pergamon Press, 1982;3:263-343.

3 Aulhorn E. Über die Beziehung Zwischen Lichtsinn und Sehschärfe. Albrecht Von Graefes Arch Ophthalmol 1964;167:4-4.

4 Johnson CA, Kelter JL, Balestrery F. Effects of target size and eccentricity on visual detection and resolution. Vis Res 1978;18:1217-22.

5 Traquair HM. Perimetry in the study of glaucoma. Trans Ophthalmol UK 1931;51:585-99.

6 Wassle H, Grunert U, Rohrenbeck J, et al. Retinal ganglion cell density and cortical magnification factor in the primate. Vis Res 1990;30:1897-911. 
7 Anderson DR. Perimetry with and without automation. 2nd ed. St Louis: CV Mosby, 1987

8 Merigan WH, Katz LM, Maunsell JHR. The effects of parvocellular lateral geniculate lesions on the acuity and contrast sensitivity of macaque monkeys. J Neuroscience 1991;11:944-1001.

9 Sample PA, Irak I, Martinez GA, et al. Asymmetries in the normal shortwavelength visual field: implications for short-wavelength automated perimetry. Am J Ophthalmol 1997;124:46-52.

10 Sample PA, Johnson CA, Haegerstrom-Portnoy G, et al. Optimum parameter for short-wavelength automated perimetry. J Glaucoma 1996:5:375-83.

11 Johnson CA, Adams AJ, Twelker JD, et al. Age-related changes in the central visual field for short-wavelength-sensitive pathways. J Opt Soc Am 1988;5:2131-9.

12 Johnson CA, Samuels SJ. Screening for glaucomatous visual field loss with frequency-doubling perimetry. Invest Ophthalmol Vis Sci 1997;38:413-25.

13 Maddess T, Severt WL. Testing for glaucoma with frequency-doubling illusion in the whole, macular and eccentric visual fields. Aust NZ J Ophthalmol 1999;27:194-6.

14 White AJ, Sun $\mathrm{H}$, Swanson WH, et al. An examination of physiological mechanisms underlying the frequency-doubling illusion. Invest Ophthalmol Vis Sci 2002;43:3590-9.

15 Anderson AJ, Johnson CA. Mechanisms isolated by frequency-doubling technology perimetry. Invest Ophthalmol Vis Sci 2002;43:398-401.

16 Kaplan E, Shapley RM. X and $Y$ cells in the lateral geniculate nucleus of macaque monkeys. J Physiol 1982;330:125-43.

17 Maddess T, Henry GH. Performance of nonlinear visual units in ocular hypertension and glaucoma. Clin Vis Sci 1992;7:371-83.

18 Livingstone MS, Hubel DH. Do the relative mapping densities of the magnoand parvocellular systems vary with eccentricity? J Neuroscience 1988;8:4334-9.
19 Jacobs NA, Patterson $\mathrm{IH}$. Variability of the hill of vision and its significance in automated perimetry. Brit J Ophthalmol 1985;69:824-6.

20 Heiil A, Lindgren G, Olsson J. Normal variability of static perimetric threshold values across the central visual field. Arch Ophthalmol 1987:105:1544-9.

21 Katz J, Sommer A. Asymmetry and variation in the normal hill of vision. Arch Ophthalmol 1986;104:65-8.

22 Parrish RK, Schiffman J, Anderson DA. Static and kinetic visual field testing reproducibility in normal volunteers. Arch Ophthalmol 1984:102:1497-502.

23 Foster PJ, Buhrmann R, Quigley HA, et al. The definition and classification of glaucoma in prevalence surveys. Br J Ophthalmol 2002;86:238-42.

24 Johnson C, Wall M, Fingeret $M$, et al. A primer for frequency doubling technology. Dublin, CA: Humphrey SystemsWelch-Allyn, Inc, 1998.

25 House P, Schulzer M, Drance $S$, et al. Characteristics of the normal central visual field measured with resolution perimetry. Graefes Arch Clin Exp Ophthalmol 1991;229:8-12.

26 Wall $M$, Chauhan $B$, Frisén $L$, et al. Visual field of high-pass resolution perimetry in normal subjects. J Glaucoma 2004;13:15-21.

27 Wojciechowski R, Trick GL, Steinman SB. Topography of the age-related decline in motion sensitivity. Opt Vis Sci 1995;72:67-74.

28 De Monasterio FM, Gouras P. Functional properties of ganglion cells of the rhesus monkey retina. J Physiol 1975;251:167-95.

29 Connolly M, Van Essen D. The representation of the visual field in parvicellular and magnocellular layers in the lateral geniculate nucleus in the macaque monkey. J Comp Neurol 1984;226:544-64.

30 Wild JM, Cubbidge RP, Pacey IE, et al. Statistical aspects of the normal visual field in short-wavelength automated perimetry. Invest Ophthalmol Vis Sci 1998;39:54-63.

31 Adams CW, Bullimore MA, Wall M, et al. Normal aging effects for frequency doubling technology perimetry. Opt Vis Sci 1999;76:582-7. 